\title{
Does Radiofrequency Ablation Stimulate a Higher Malignant Potential of Hepatocellular Carcinoma?
}

\author{
Akiyoshi Kinoshita ${ }^{1 *}$, Hirokazu Nishino ${ }^{1}$ and Hisao Tajiri ${ }^{2}$ \\ ${ }^{1}$ Division of Gastroenterology and Hepatology, The Jikei University Daisan Hospital, Japan \\ ${ }^{2}$ Division of Gastroenterology and Hepatology, Department of Internal Medicine, The Jikei University School of Medicine, Japan
}

\begin{abstract}
Received: December 21, 2013; Accepted: January 03, 2014; Published: January 08, 2014
"Corresponding author: Akiyoshi Kinoshita, Division of Gastroenterology and Hepatology, The Jikei University Daisan Hospital, 4-11-1 Izumihon-cho, Komae- shi, Tokyo, 201-8601, Japan, Tel: 03-3480-1151; Fax: 03-3480-6688; Email: aki.kino@jikei.ac.jp
\end{abstract}

Radiofrequency ablation (RFA) is an established treatment for BCLC 0-A hepatocellular carcinoma (HCC) associated with minimal invasiveness and a high rate of curability [1]. Recent large scale, long-term follow-up study from Korea demonstrated the 5 and 10 year overall survival rates in 1,305 patients with early HCC treated with RFA to be $59.7 \%$ and $32.3 \%$ respectively $[2]$.

However, to date, it remains under debate which is superior, surgical resection or RFA, with respect to the overall survival and recurrence rates in patients with early HCC $[3,4]$. A major drawback of RFA is the high rate of tumor recurrence after the therapeutic procedure, which affects the patient survival and is the main reason why RFA is considered to be inferior to surgical resection in cases of resectable lesions [5]. In the aforementioned study from Korea, Kim et al. demonstrated the 5 and 10 year cumulative local tumor recurrence rates to be $27.0 \%$ and $36.9 \%$ respectively, with 5 and 10 year cumulative intrahepatic distant and extrahepatic recurrence rates of $73.1 \% / 88.5 \%$ and $19.1 \% /$ $38.2 \%$ respectively [2].

The presence of an insufficiently ablated margin, in addition to a large tumor size, is a major risk factor for tumor recurrence after RFA. Lee et al. reported that an insufficiently ablated margin is an independent factor predicting early diffuse recurrence (three or more new lesions of intrahepatic recurrence within one year after the initial RFA procedure) [6]. Some investigators have reported early and serious recurrence of HCC after RFA, leading to poor survival outcomes $[7,8]$. These reports suggest the phenotypic and functional alteration of HCC cells after RFA.

More recently, in vol. 58 issue 5 of Hepatology, Yoshida et al. demonstrated that sublethal heat treatment of HCC cells endows these cells with a higher proliferative and carcinogenic potential in both in vitro and in vivo studies [9]. The authors exposed three human HCC cell lines (HEPG2, HuH7 and HEP3B) to $37^{\circ} \mathrm{C}$ $45^{\circ} \mathrm{C}, 50^{\circ} \mathrm{C}$ and $55^{\circ} \mathrm{C}$ for 10 minutes, simulating the marginal zone of RFA treatment. They subsequently analyzed the degree of cell proliferation, parameters of the epithelial-mesenchymal transition (EMT) and the level of activation of mitogen-activated protein kinases 5-12 days after treatment. Although all HCC cells died following exposure to a temperature of $55^{\circ} \mathrm{C}, 65-85 \%$ of the HCC cells survived exposure to temperature of $48-50^{\circ} \mathrm{C}$, thus exhibiting distinct morphological changes, such as the appearance of spindle-like cells, and expressing the progenitor marker, CD133, the cholangiocyte markers, CK7 and CK19, and the EMT-related transcription factor, Snail, on day 5 after heat exposure. The HCC cells exposed to $50^{\circ} \mathrm{C}$ showed enhanced proliferation and activation of Src homology and collagen (Shc), a central player in malignant transformation. Moreover, in order to evaluate how far heat treatment can indue more aggressive (EMT-like) tumor growth in vivo, the authors transplanted heat-exposed HEPG2 cells into nude mice. The heat-exposed HEPG2 cells implanted into the nude mice induced the growth of significantly larger, more aggressive tumors than the untreated cells, as well as enhanced proliferation and the upregulation of various markers of the EMT. The authors concluded that insufficient or sublethal heat treatment stimulates a higher malignant potential by skewing HCC cells toward the EMT and transforming them into a progenitor-like, highly proliferative cellular phenotype both in vitro and in vivo.

The above study supports the presence of early and serious recurrence leading to unfavorable outcomes after RFA [6-8] and stresses the need to apply sufficiently high temperatures and obtain wide therapeutic margins. Clinicians should therefore recognize the possibility of malignant transformation of HCC cells subject to heat treatment, such as RFA. In such cases, the need for additional treatment, including surgical resection and/ or TACE, should be carefully taken into consideration.

\section{Conflict of Interest}

The authors declared that they do not have anything to disclose regarding funding or conflict of interest with respect to this manuscript.

\section{References}

1. European Association For The Study of The Liver, European Organisation For Research And Treatment Of Cancer (2012) EASLEORTC clinical practice guidelines: Management of hepatocellular carcinoma. J Hepatol 56: 908-943.

2. Kim YS, Lim HK, Rhim H, Lee MW, Choi D, et al. (2013) Ten-year 
outcomes of percutaneous radiofrequency ablation as first-line therapy of early hepatocellular carcinoma: analysis of prognostic factors. J Hepatol 58: 89-97.

3. Pompili M, Saviano A, de Matthaeis N, Cucchetti A, Ardito F, et al. (2013) Long-term effectiveness of resection and radiofrequency ablation for single hepatocellular carcinoma $\leq 3 \mathrm{~cm}$. Results of a multicenter Italian survey. J Hepatol 59: 89-97.

4. Hasegawa K, Kokudo N, Makuuchi M, Izumi N, Ichida T, Kudo M, et al. (2013) Comparison of resection and ablation for hepatocellular carcinoma: a cohort study based on a Japanese nationwide survey. J Hepatol 58: 724-729.

5. Tiong L, Maddern GJ (2011) Systematic review and meta-analysis of survival and disease recurrence after radiofrequency ablation for hepatocellular carcinoma. Br J Surg 98: 1210-1224.
6. Lee HY, Rhim H, Lee MW, Kim YS, Choi D, et al. (2013) Early diffuse recurrence of hepatocellular carcinoma after percutaneous radiofrequency ablation: analysis of risk factors. Eur Radiol 23: 190197.

7. Portolani N, Tiberio GA, Ronconi M, Coniglio A, Ghidoni S, et al. (2003) Aggressive recurrence after radiofrequency ablation of liver neoplasms. Hepatogastroenterology 50: 2179-2184.

8. Ruzzenente A, Manzoni GD, Molfetta M, Pachera S, Genco B, et al. (2004) Rapid progression of hepatocellular carcinoma after Radiofrequency Ablation. World J Gastroenterol 10: 1137-1140.

9. Yoshida S, Kornek M, Ikenaga N, Schmelzle M, Masuzaki R, et al. (2013) Sublethal heat treatment promotes epithelial-mesenchymal transition and enhances the malignant potential of hepatocellular carcinoma. Hepatology doi: 10.1002/hep.26526. 